\title{
Apocrine Gland Anal Sac Adenocarcinoma in Dogs: 22 Cases (2015-2020)
}

\author{
A.K. Sahoo', I. Nath², S.B. Senapati ${ }^{3}$, S.K. Panda', M.R. Das', B.K. Patra ${ }^{1}$
}

10.18805/IJAR.B-4371

\begin{abstract}
Background: Apocrine gland anal sac adenocarcinoma (AGASACA) is a highly malignant disease mostly invading regional lymph nodes at the time of disease diagnosis.

Methods: Twenty- two cases of Apocrine gland anal sac adenocarcinoma (AGASACA) were treated during five years. Contrast radiography with lohexol and Lipidol were carried out to detect primary anal sac mass and regional lymph node through indirect lymphography technique. For clinical staging, ultrasonography, fine-needle aspiration cytology, histopathology of both primary tumor mass and regional lymph node were performed. Closed anal Sacculectomy was performed as curative therapy for anal sac adenocarcinoma.

Result: The median age and weight of dogs at presentation were 8.4 years and $20.9 \mathrm{~kg}$, respectively. Diagnostic radiology, histopathology and biochemical assay confirm twenty-two cases of anal sac adenocarcinoma and medial iliac lymph node to be sentinel lymph node. Carcinoma was of unilateral occurrence in all the dogs. Four (18\%) dogs had hypercalcemia and twelve (54\%) had metastases to the regional lymph nodes with clinical signs of tenesmus or constipation, polyuria -polydipsia and anorexia. Apocrine gland Adenocarcinoma of anal sac was found to initially metastasize to the medial iliac lymph node. Survival times of dogs treated with the surgical procedure only (8 out of 22 dogs) were appreciably longer than the dogs with therapeutic only. Animals with metastasis to regional lymph nodes or lymphadenopathy had poor prognosis.
\end{abstract}

Key words: Anal Sacculectomy, Iohexol, Indirect lymphography, Lipidol, Lymphadenectomy.

\section{INTRODUCTION}

Anal sacs or Anal glands are hemispherical modified skin structure located at 4 O'Clock and 8 O'Clock position laterally to the anus, lined by stratified squamous epithelium, which secrets the contents of apocrine glands into the tubular portion (Jung et al., 2016; Kokila et al., 2016). Diseases of para anal sinuses or anal sac are common in canines, with prevalence ranging from 2 to $12 \%$ of all skin tumors (Hobson et al., 2006). AGASACA are highly malignant neoplasms that obliterate the anal sac, readily invade the surrounding soft tissue even when small in size and frequently metastasize to the regional lymph nodes $(90 \%)$ i.e., medial iliac lymph node and to visceral organs like lungs, liver and spleen (Meuten et al., 1981; Goldschmidt and Zoltowski, 1981; William et al., 2003) and rarely to other organs (Morris and McNaugh, 2015). Anal sac tumors usually occur unilaterally, with the initial diagnosis made through digital rectal palpation (Morris and McNaugh, 2015). A significant proportion of animals develop systemic clinical signs with polyuria, polydipsia, weakness, vomiting and anorexia due to hypercalcemia of malignancy resulting from the secretion of Parathyroid hormone-related protein (PTHrP) by the neoplastic cells (Williams et al., 2003; Lucas et al., 2007). The clinical staging involving Tumor Node Metastasis (TNM) classification of canine AGASACA is same as that of skin and adnexa, categorizing it into four stages (stage I, II, Illa, Illb, IV) ( Polton and Brearley, 2007). Histopathologically there are three subtypes of AGASACA viz., solid type, rosette type and tubular types (Meuten et al., 1981). The treatment
${ }^{1}$ Teaching Veterinary Clinical Complex, College of Veterinary Science and Animal Husbandry, Odisha University of Agriculture and Technology, Odisha, India.

'Department of Veterinary Surgery and Radiology, College of Veterinary Science and Animal Husbandry, Odisha University of Agriculture and Technology, Odisha, India.

${ }^{3}$ Institute of Life Sciences, Tumor Microenvironment and Animal Models Lab, Nalco Square, Bhubaneswar-751 023, India.

${ }^{4}$ Department of Veterinary Pathology, College of Veterinary Science and Animal Husbandry, Odisha University of Agriculture and Technology, Odisha, India.

Corresponding Author: A.K. Sahoo, Teaching Veterinary Clinical Complex, College of Veterinary Science and Animal Husbandry, Odisha University of Agriculture and Technology, Bhubaneswar751 003, Odisha, India. Email: aks76vet@gmail.com

How to cite this article: Sahoo, A.K., Nath, I., Senapati, S.B., Panda, S.K., Das, M.R., Patra, B.K. (2021). Apocrine Gland Anal Sac Adenocarcinoma in Dogs: 22 Cases (2015-2020). Indian Journal of Animal Research. DOI: 10.18805/IJAR.B-4371.

Submitted: 30-11-2020 Accepted: 01-04-2021 Online: 29-04-2021

of AGASACA mainly involves surgery (local, with or without resection of metastatic lymph nodes), chemotherapy and radiation therapy depending upon the tumor stages and condition of the animal (Hobson et al., 2006).

The purpose of the present study was to evaluate clinical symptoms, fine needle aspiration cytology of tumor mass, indirect lymphography technique through tracer injection for detection of regional lymph node metastasis, 
abdominal ultrasonography for possible metastasis, blood biochemical analysis for systemic changes and adopting a particular surgical procedure for successful clinical therapy.

\section{MATERIALS AND MeTHODS}

The study was conducted in the Department of Teaching Veterinary Clinical Complex and Department of Veterinary Surgery and Radiology, College of Veterinary Science and Animal Husbandry, OUAT, Bhubaneswar from 2015-2020 for five years. During this period, fifty-five dogs were examined for any of the diseases of perianal region. Thirtythree dogs treated successfully with therapeutics were categorized into four groups basing on clinical symptoms such as anal sacculitis (15 no.), anal sac abscesses (8 no.), anal sac fistula or furunculosis (5 no.), perianal adenoma (5 no.) and hence excluded from the present study population. Rest, twenty-two dogs that were diagnosed as apocrine gland adenocarcinoma of anal sac form the subjects of the present study. These dogs showed the following clinical symptoms like anorexia, perianal swelling, tenesmus or dyschezia, fecal incontinence, scooting, dragging or rubbing their hind end on the ground, licking or chewing their anal or tail-base regions, polyuria, polydipsia and lethargy. Swollen mass with hard consistency was palpable on per rectal examination through fingertip during anal sac expression.

\section{Diagnostic imaging}

Survey radiography of abdomen and pelvis (right lateral, left lateral, ventrodorsal) with and without contrast study of the anal sac mass was performed. $2 \mathrm{ml}$ of lohexol (watersoluble iodinated contrast agent, lohexol Injection USP, 300 $\mathrm{mg} \mathrm{I/mL;} \mathrm{Omnipaque} \mathrm{TM}^{\mathrm{M}}$ 300; GE Healthcare, Ireland) was injected using a 24 G IV catheter or 24G hypodermic needle into the anal sac through the anal sac duct until it was full and overflowing (Fig 1 ) followed by radiography of individual and both anal sacs at intervals of $3,5,10,20,60$ minutes and 24 hours of the injection. $2 \mathrm{ml}$ of Lipidol Ultrafluid (Ethyl ester of iodized fatty acids of poppy seed oil with lodine content $48 \%$ i.e., $480 \mathrm{mgl} / \mathrm{ml}$, Lipidol Ultra fluid, Guerbet, France) was injected subcutaneously and intradermally through the indirect lymphographic technique with a 24 gauze needle both on the dorsal-sacral space and near popliteal lymph node. Serial radiographs were taken immediately after the injection, followed by 5, 10, 15, 30, 60 and 120-min and 24 hours after injection. Routine grayscale ultrasonography (Ultrasound machine, GE Healthcare, Ireland), elastography and color flow Doppler of the anal sac and regional lymph node were performed with a convex transducer $(8 \mathrm{MHz})$.

Clinical staging of twenty-two (22) cases of anal sac apocrine gland adenocarcinoma was done basing on tumor size, regional lymph node size, fine needle aspiration cytology of the tumor mass and medial iliac lymph node through ultrasound-guided aspiration and three-view thoracic radiography following the guidelines of Clinical staging scheme by Polton and Brearley, 2007. Complete blood count and serum biochemistry (including ionized calcium level) were also performed to assess for the presence of concurrent or paraneoplastic syndrome.

\section{Surgery technique (Closed technique)}

Unilateral closed anal sacculectomy was performed in eight dogs under general anesthesia out of twenty-two dogs. A curvilinear incision was given using a Metzenbaum scissor separating the sac's outer part from the internal, external and sphincter muscles surrounding it. The duct, along with anal sac mass was excised from mucocutaneous junction by ligating with a 2-0 PDS (polydioxanone) and skin was closed with 2-0 Nylon sutures. Postoperatively, the dogs were administered with antibiotics (Ceftriaxone @ 20mg/ $\mathrm{kg}$ ), Injection Meloxicam (0.2-0.4 mg/kg)and Metronidazole @ $15 \mathrm{mg} / \mathrm{kg}$ body wt for at least 5 days and laxatives (Psyllium or Lactulose). Tissue specimens were fixed in $10 \%$ formalin, processed routinely, embedded on paraffin, sectioned in $5 \mu \mathrm{m}$ and stained with hematoxylin-eosin ( $\mathrm{H}$ and $\mathrm{E})$.

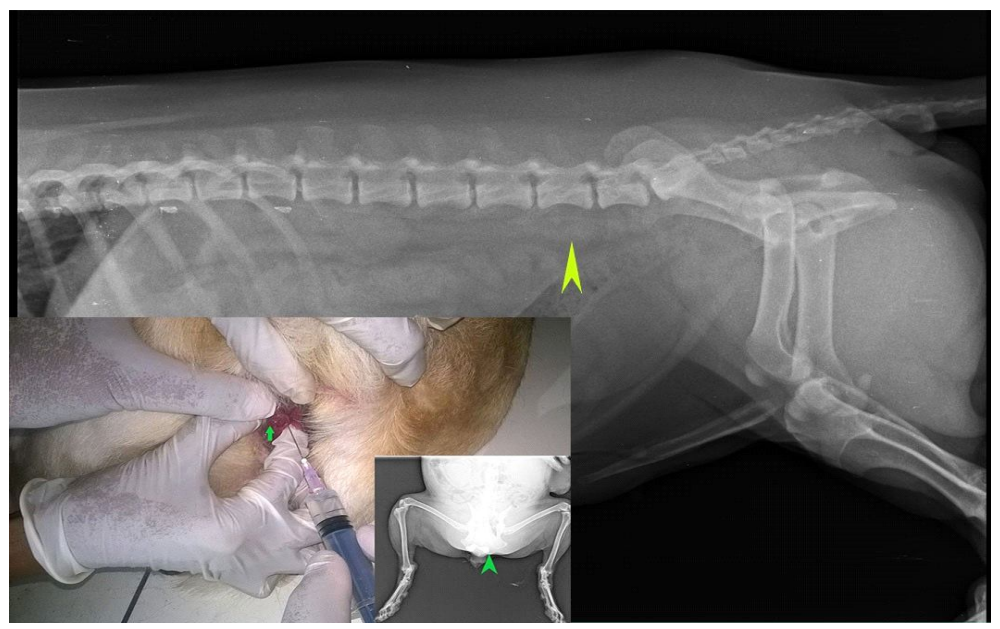

Fig 1: Survey radiography of abdomen with colored arrow head indicating enlarged sacroiliac lymph nodes. Inset- Iohexol (Omnipaque) injection into both the anal sac in a Labrador, female and radiography immediately after injection (5 minutes). 


\section{RESULTS AND DISCUSSION}

Twenty-two dogs from common breeds of dogs like Labrador, German shepherd, Doberman, Dachshund, Spitz were examined with the median age at diagnosis 8.4 years (range 2-15 years) and median weight $20.9 \mathrm{~kg}$ (range 7-35 kg). The male and female ratio during clinical presentation was in equal proportion i.e. 11 male to 11 female. Symptoms of hypercalcemia (serum calcium $12-15 \mathrm{mg} / \mathrm{dL}$ ) like polyuria, polydipsia and lethargy were observed in four dogs which were controlled with prednisone $(1.5 \mathrm{mg} / \mathrm{kg}$, orally, every 6 hours), but the owners were very reluctant for any surgical intervention and these dogs were lost to follow up.

\section{Diagnostic imaging}

Abdominal and three views thoracic radiography did not reveal metastasis to any visceral organs such as liver, spleen, spinal cord, or thorax in the present study. lohexol injection clearly outlined anal sac proper in eight animals $(8 / 22)$ (Fig 1) with the closure of anal sac duct in two dogs
(2/22) and constricted anal sac in three dogs (3/22). This helps in differentiating AGASACA from other anal sac diseases like anal sac fistula or furunculosis, where iohexol will flow through the fistulous tract. With Lipidol ultra-fluid injection (Fig), the lymphatic plexus of the hind limb, efferent lymphatic vessels, superficial inguinal lymph node (Si LN), medial-iliac lymph nodes (mi LN) and popliteal lymph nodes (Po LN) were visualized with radiography in different time setting. Ultrasonography and elastography of the anal sac tumor mass were performed in 6 animals depicting hardened and enlarged anal sac gland (Fig 3). Doppler ultrasonography (Fig 4) of medial iliac lymph nodes showed heterogeneous echo pattern and length $2.00 \mathrm{~cm}$ with $\mathrm{S} / \mathrm{L}$ ratio of 0.52 . On elastography, the medial-iliac lymph node was seen to constricting the passage of the large bowel and measured $3.46 \mathrm{~cm}$. Enlarged lymph node size was the primary marker of metastasis (Llabrés-Díaz, 2004; De Swarte et al., 2011) rather than other changes in lymph node shape or echogenicity as found in our study.

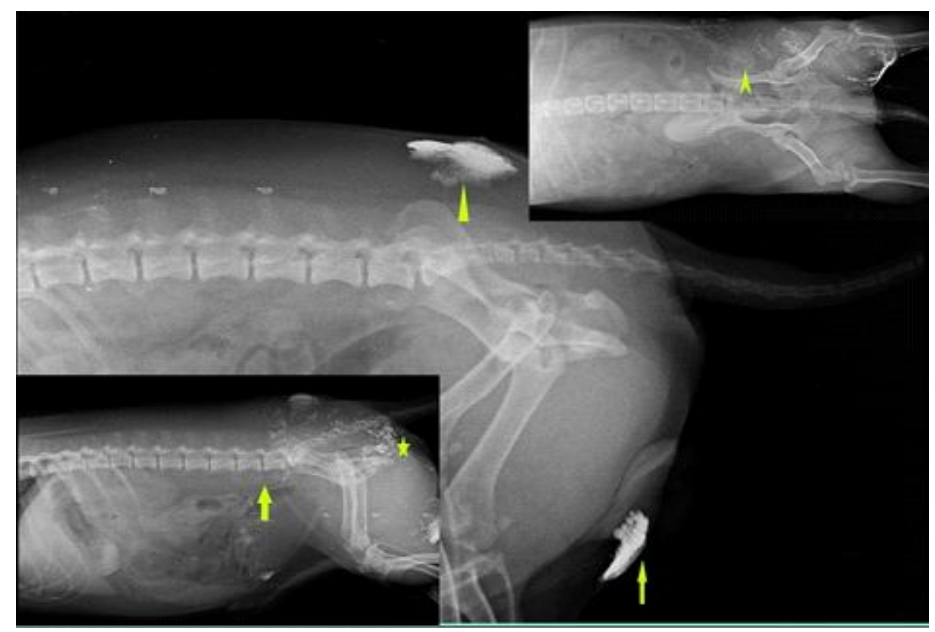

Fig 2: Lipidol ultrafluid injection intradermally at dorsal sacral space and near popliteal LN area. Radiography immediately after injection, $48 \mathrm{hr}$ and $72 \mathrm{hr}$ post injection.

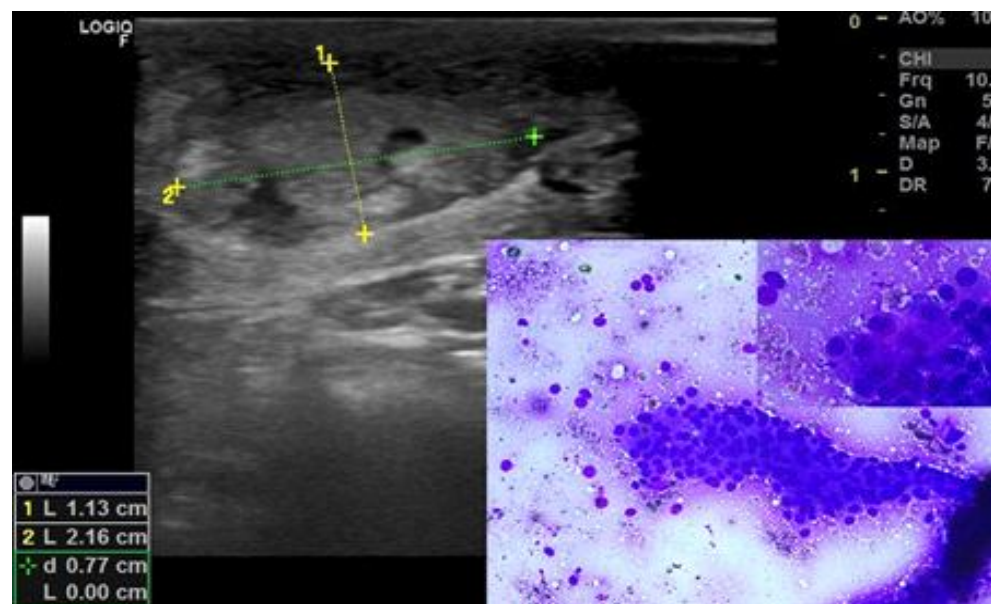

Fig 3: USG of the anal sac mass with dog in lateral position.

Inset- photomicrography of FNAC of the anal sac mass with cohesive population of hepatoid cells. Magnification in $40 \mathrm{X}$ and $100 \mathrm{X}$. 


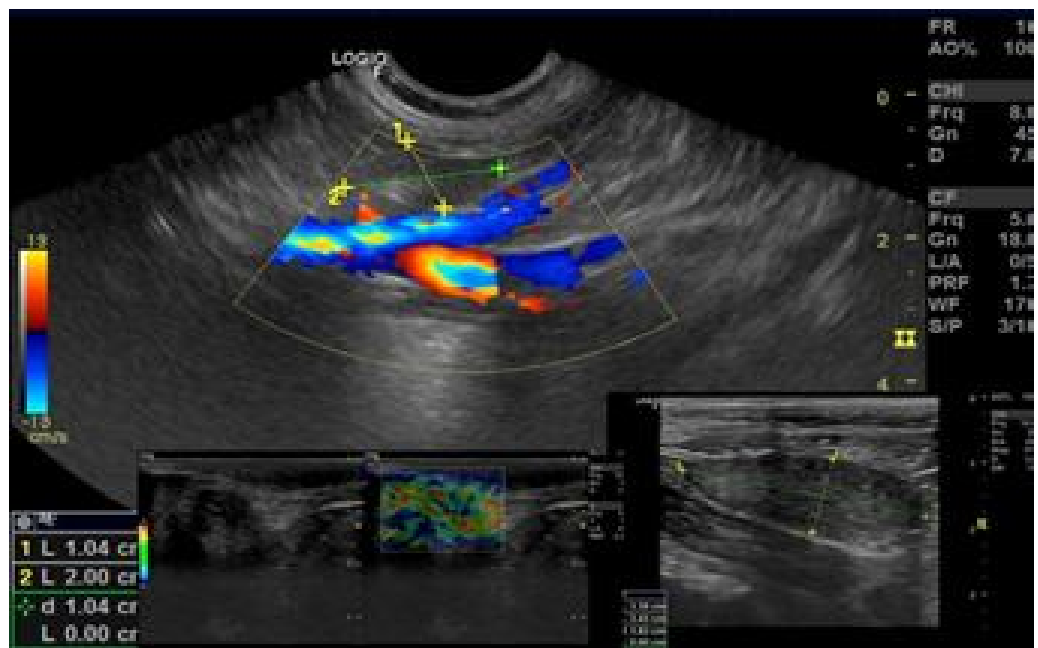

Fig 4: USG of enlarged ileac LN and sacroiliac lymphocentre along with elastography and color flow to detect the toughness (hardness) of lymph node.

\section{Clinical staging}

Primary tumor mass at initial patient presentation was recorded in eighteen dogs with sizes ranging from $0.4 \mathrm{~cm}$ to $6 \mathrm{~cm}$. In the rest four dogs, tumor size could not be recorded as these were lost to follow up. Lymph nodes were measured in thirteen dogs, out of which enlarged lymph nodes with sizes ranged from $0.8 \mathrm{~cm}$ to $5 \mathrm{~cm}$ were documented in six dogs. So, twelve dogs were found in stage I, four dogs were in stage II, six dogs were in stage IIIa and none of the dogs studied were in stage IIllb or stage IV.

Fine needle aspirates of the anal sac mass were performed in eight dogs for confirmation of malignancy i.e. anal sac adenocarcinoma. Microscopic examination of FNAC (Fig 3) revealed trabecular to circular clusters of cuboidal epithelial cells with medium blue cytoplasm. Cell borders are somewhat distinct and nuclei were more paracentral to basal in these cuboidal to low columnar cells that were typical for the classic apocrine anal sac adenocarcinoma. In lymph node aspirate (medial iliac lymph node and popliteal lymph node), characteristic malignant cell types of cuboidal cell types, bluish cytoplasm with distinct border and large central or paracentral nucleus were found indicating metastasis (Fig 5).

\section{Surgical findings and postoperative care}

Primary anal sac mass was removed through closed anal sacculectomy (Fig 6) in eight dogs in stage I out of twelve dogs. Surgical margins were narrow (less than $5 \mathrm{~mm}$ ) in five dogs and incomplete (tumor cells in contact with margin) in three dogs. Eight dogs that underwent surgical procedures survived longer than the rest fourteen dogs in which surgery was not performed. There were recurrence and progression of tumor in four dogs after eight months, two of which were euthanized with owner's consent. Among the fourteen dogs in non-surgical group, six dogs were lost to follow up after four months, four dogs died for unknown reasons and rest four dogs lived up to one year. Histopathological examination (Fig 7) of the excised biopsy revealed mostly solid pattern

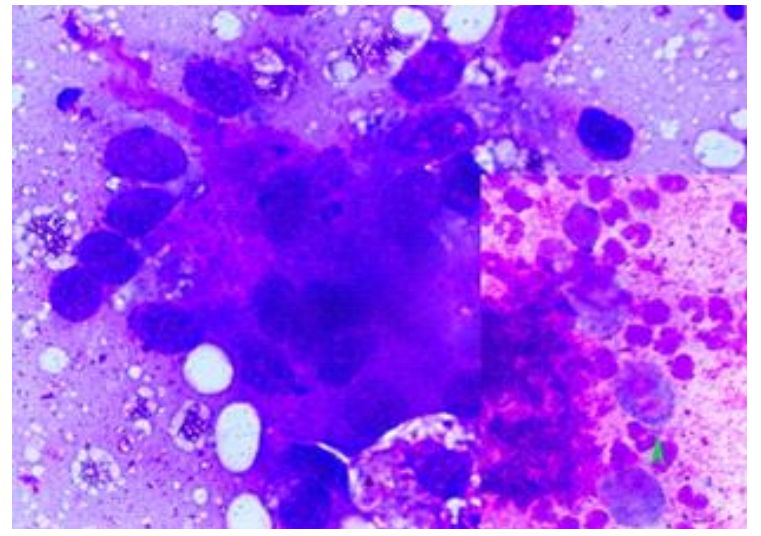

Fig 5: Photomicrography of FNAC of the medial iliac lymph node. Inset- FNAC of popliteal lymph node. Both seen under high power (100X) with characterstic neoplastic cells.

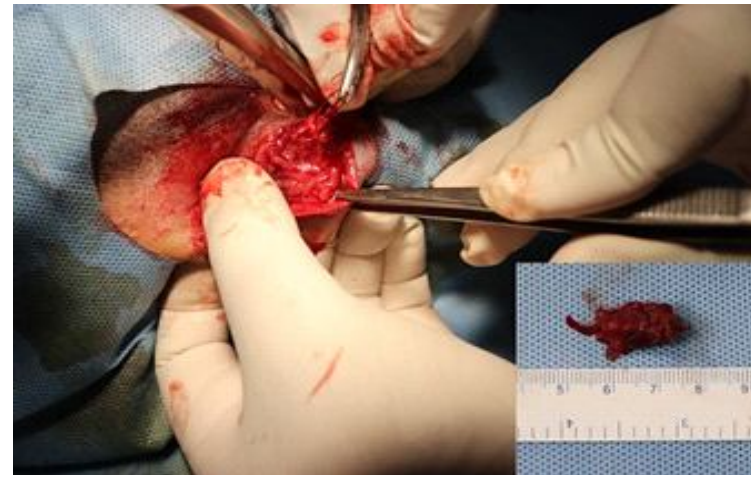

Fig 6: Closed anal sacculectomy of the left anal sac in a dachshund. Inset- Excised anal sac tumor mass.

with vascular invasion with sheets of oval to round-shaped pleomorphic neoplastic cells with characteristic hyperchromatic nuclei, eosinophilic cytoplasm, occasional anisocytosis, anisokaryosis, numerous mitotic figure and without any glandular differentiation. 


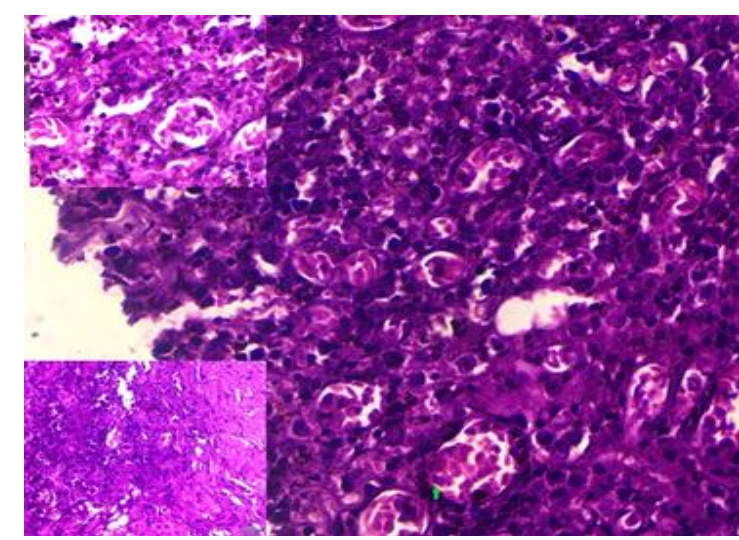

Fig 7: Histopathology of the excised anal sac detecting non glandular solid pattern of adenocarcinoma of apocrine gland.

AGASACA are reported to be locally aggressive tumor that infiltrates into anal sphincter muscle and surrounding tissues and frequently metastasize early. The mean survival times of 8 dogs of the Surgery group studied in this case series of 22 dogs were appreciably longer ( $>$ one and a half year or 500 days) with a low morbidity rate as compared to the rest fourteen dogs (with survival time < one year or 365 days) in which surgery could not be performed due to various reasons which are in consistent to earlier reports of William et al., 2003 and Barnes andDemetriou, 2017. Greater chances of survival in surgical group means earlier diagnosis and choosing an appropriate method of surgical intervention as a first-line treatment when the carcinoma is in stage I, II and Illa that is primary tumor mass of all sizes with initial draining to regional lymph nodes, similar to earlier reports of Barnes and Demetriou, 2017 and Hobson et al., 2006. In this case study, we choose several diagnostic methods to have more sensitivity and specificity for detection of AGASACA and report eight cases of AGASACA in which closed anal sacculectomy were performed as first-line treatment. Surgical margins are a significant factor in tumor resection as wide margins involving anal sphincter muscles lead to fecal incontinence (1/8) or incomplete margins removal leads to tumor recurrence (2/8); the later two dogs were subsequently euthanized as per the owner's consent. The most common breeds of dogs affected in this series were all purebred dogs, similar to findings by Polton et al., 2006 but inconsistent with earlier reports of greater percentage involvement of mixed breed dogs (Goldschmidt and Schofer, 1992). Though gender predisposition seems to be a conflicting report with most recent studies (Polton et al., 2006), we found almost $50 \%$ prevalence of AGASACA in male dogs, which is inconsistent with findings of Williams et al.,2003. AGASCA has been detected both in intact and neutered male and female dogs (17 intact, 4 neutered and 1 not recorded) differing from earlier reports of Polton et al., 2006 with a significant risk of occurrence in male neutered dogs to be 1.4. Again, the mean age at presentation was 8.4 years, emphasizing the percentage of occurrence, more in older animals. Neutering did not affect much to the occurrence of AGASACA in older males as the percentage of presentation is only $18 \%(4 / 22)$. Therefore, careful palpation of anal sacs and perianal area with the expression of anal sacs contents may be routinely practiced in older dogs of either sex during routine physical examination.

Anal sac mass was mostly unilateral on physical examination. Bilateral occurrence is occasional (Morris and Mc Naugh, 2015); however, it had not been reported in this case series which is in consistent with findings of Williams et al., 2003. Common clinical symptoms like dyschezia, scooting, licking the anal region were observed in almost all animals (Morris and Mc Naugh, 2015). In additions polyuria, polydipsia and lethargy were the most common signs in four dogs $(4 / 22)$ i.e. approximately $18 \%$ in which serum calcium estimation was confirmed to be due to hypercalcemia ( Meuten et al., 1981; Williams et al., 2003; Barnes and Demetriou, 2017). The hypercalcemia was controlled in one dog with prednisone $(1.5 \mathrm{mg} / \mathrm{kg}$ orally every 6 hours) for two months. Prednisone was chosen to promote the excretion of calcium in the urine, reduce intestinal calcium absorption and inhibit calcium resorption from the bone. Dogs detected with hypercalcemia associated with AGASACA had shorter survival times than dogs that were normocalcemic $(<9.5 \mathrm{mg} / \mathrm{dl})$.

Detection of enlarged lymph nodes and identification of medial iliac lymph nodes were done through indirect lymphography technique for identification of sentinel lymph nodes in AGASACA (Majeski et al., 2017; Linden et al., 2018). Sentinel lymph node is the initial lymph node to which metastasis spread from the primary tumor mass. Medial iliac lymph nodes were the sentinel lymph nodes for AGASACA as reported through the indirect CT lymphography technique of Majeski et al., 2017 and radiocolloid lymphoscintigraphy procedure of Linden et al., 2018. In the present study, Lipidol ultra-fluid (oil-based contrast agent) has been used for detection of medial iliac lymph nodes for the first time in AGASACA cases of dog with partial modification to the technique. Instead of injecting tracer to the primary tumor, it has been injected above the dorsal sacral space and near the popliteal lymph node for confirming the actual lymphatic pathway. The resulting lymphatic plexus of the hind limb was detected through the subcutaneous and intradermal injection of oil-based tracer. The lymphatic channel originates from the anal sac tumor to medial iliac lymph nodes (mi LN) to superficial and deep inguinal lymph nodes, then to popliteal lymph nodes. Hence, fine needle aspiration of medial iliac lymph node and popliteal lymph nodes shows characteristic features of malignancy. Further, malignancy of the sentinel lymph node that is the medial iliac lymph node was detected through greyscale ultrasonography along with color flow and elastography (llabre' s-di' az et al., 2004, De Swarte et al., 2011). lohexol was injected as a watersoluble tracer to outline the shape of anal sac and primary tumor mass (Jung et al., 2016), simultaneously differentiating from other conditions like anal sac fistula, sinuses. Enlarged lymph node and primary tumor mass were also confirmed 
through digital rectal palpation. However, there was no evidence of metastasis to the thorax or other visceral organs as seen through three views thoracic X-ray. Though abdominal radiographs were sufficient to see evidence of advanced disease and regional lymph node enlargement but abdominal ultrasound was superior for evaluating regional lymphadenomegaly (Polton and Brearly, 2007). Lymph node malignancy was confirmed through FNA cytology as per the reports of Fournier et al., 2018 for the staging of malignant solid tumor and detection of multiple nodal metastases.

\section{CONCLUSION}

In summary, early diagnosis of apocrine gland anal sac adenocarcinoma through use of simple diagnostic modalities like tracer (oil-based and water-soluble) for lymphographic study, ultrasonography, fine needle aspiration cytology and biochemical estimation will help in detecting the disease before it spreads to regional lymph nodes and become metastatic. Prompt surgical intervention will prevent the distant spread of the disease, thereby increasing the survival time and conditions of life. A longer period of remission can be achieved through larger histologic margins during surgical removal.

\section{REFERENCES}

Barnes, D.C., Demetriou, J.L. (2017). Surgical management of primary, metastatic and recurrent anal sac adenocarcinoma in the dog: 52 cases. J of Small Animal Practice. DOI: 10.1111 /jsap. 12633 .

De Swarte, M., Alexander, K., Rannou, B., D’Anjou, M.A., Blond, L. and Beauchamp, G. (2011). Comparison of sonographic features of benign and neoplastic deep lymph nodes in dogs. Vet Radiol Ultrasound. 52(4): 451-6. doi: 10.1111/ j.1740-8261.2011.01808.x.

Fournier, Q., Cazzini, P., Bavcar, S., Pecceu, E., Ballber, C., Elders, R. (2018). Investigation of the utility of lymph node fineneedle aspiration cytology for the staging of malignant solid tumors in dogs. Veterinary Clinical Pathology. 47: 489-500. https://doi.org/10. 1111/vcp.12636.

Goldschmidt, M.H. and Zoltowski, C. (1981). Anal sac adenocarcinomas in the dog: 14 cases. J Small Anim Practice. 22: 119-128

Hobson, H.P., Brown, M.R. and Rogers, K.S. (2006). Surgery of Metastatic Anal Sac Adenocarcinoma in Five Dogs. Veterinary Surgery. 35: 267-270.

Jung, Y., Jeong, E., Park, S., Jeong, J., Choi, U.S., Kim, M.S., Kim, N. and Lee, K. (2016). Diagnostic imaging features of normal anal sacs in dogs and cats. Journal of Veterinary Science. 17(3): 331-335.
Kokila, S., Veena, P., Kumar, R.V.S. and Srilatha, Ch. (2016). Clinical studies on anal tumors in dogs. Indian Journal of Animal Research. 50(4): 629-631.

Linden, D.S., Cole, R., Tillson, D.M., Boothe, H.W., Matz, B.M. (2019). Sentinel lymph node mapping of the canine anal sac using lymphoscintigraphy: A pilot study. Vet Radiol Ultrasound. 60: 346-350. https://doi.org/10.1111/vru. 12707.

Llabrés-Díaz, F.J. (2004). Ultrasonography of the medial iliac lymph nodes in the dog. Veterinary Radiology and Ultrasound. 45(2): 156-165.

Lucas, P., Lacoste, H., de Lorimier, L.P. and Fan, T.M. (2007). Treating paraneoplastic hypercalcemia in dogs and cats. Vet Med. 102: 314-331.

Majeski, S.A., Steffey, M.A., Fuller, M., Hunt G.B., Mayhew, P.D., Pollard, R.E. (2017). Indirect computed Tomographic lymphography for iliosacral lymphatic mapping in a cohort of dogs with anal sac gland adenocarcinoma: Technique description. Vet. Radiology Ultrasound. 0(0): 1-9.

Meuten, D.J., Cooper, B.J., Capen, C.C., Chew, D.J.and Kociba, G.J. (1981). Hypercalcemia associated with an adenocarcinoma derived from the apocrine glands of the anal sac. Vet Pathology. 18: 454-471.

Morris, J. and McNaugh, K. (2015). Anal Sac Adenocarcinoma; A Hidden Cancer. International Animal Health Journal. 2(4): 38-43. Corpus ID: 20981207.

Polton, G.A. and Brearley, M.J. (2007). Clinical stage, therapy and prognosis in canine anal sac gland carcinoma. Journal of Veterinary Internal Medicine. 21: 274-280.

Polton, G.A., Mowat, V., Lee, H.C., Mckee, K.A. and Scase, T.J. (2006). Breed, gender and neutering status of British dogs with anal sac gland carcinoma. Veterinary and Comparative Oncology. 4(3): 125-131.

Williams, L.E., Gliatto, J.M.and Dodgo, R.K. (2003). Carcinoma of the apocrine glands of the anal sac in dogs: 113 cases (1985-1995). Journal of American Veterinary Medical Association. 223(6): 825.

Linden, D.S., Cole, R., Tillson, D.M., Boothe, H.W., Matz, B.M. (2018). Sentinel lymph node mapping of the canine anal sac using lymphoscintigraphy: A pilot study. Vet Radiol Ultrasound: 1-5. https:// doi.org/10.1111/vru.12707.

Llabrés-Díaz, F.J. (2004). Ultrasonography of the medial iliac lymph nodes in the dog. Veterinary Radiology and Ultrasound. 45(2): 156-165.

Goldschmidt MH, Schofer FS (1992). Anal sac gland tumors. In: Skin tumours of the dog and cat. 1st edn. Pergamon Press, Oxford,UK, :103-108. 